\title{
QoT-Aware Optical Amplifier Placement in Filterless Metro Networks
}

\author{
Memedhe Ibrahimi ${ }^{1 *}$, Omran Ayoub ${ }^{1}$, Oleg Karandin ${ }^{1}$, Francesco Musumeci $^{1}$, Andrea Castoldi ${ }^{2}$, \\ Rosanna Pastorelli ${ }^{2}$, Massimo Tornatore ${ }^{1}$ \\ ${ }^{1}$ Department of Electronics, Information and Bioengineering, Politecnico di Milano, Milan, Italy \\ ${ }^{2}$ SM Optics, Vimercate (MB), Italy \\ ${ }^{*}$ Corresponding author: memedhe.ibrahimi@ polimi.it
}

\begin{abstract}
Filterless Optical Networks (FONs) represent a novel cost-effective solution for metro optical networks, that allows to achieve equipment-cost savings by removing expensive optical-switching components from network nodes. In this study, we investigate how to further reduce equipment cost in FONs by minimizing amplifiers' cost. We propose a Genetic Algorithm (GA) for placing boosters, inline amplifiers and pre-amplifiers in FONs with the objective of minimizing amplifiers cost. We provide two versions of the GA and compare their performance against a baseline amplifier placement in terms of amplifiers cost and quality-of-transmission (QoT), i.e., lightpaths OSNR and received power. Moreover, we provide a comparison between filterless and wavelength-switched architectures. Simulative results achieved over realistic network topologies show significant amplifier cost savings, up to $60 \%$ compared to baseline approaches.

Index Terms-Filterless Optical Networks, Optical Amplifiers, Cross-layer Design
\end{abstract}

\section{INTRODUCTION}

Filterless Optical Networks (FONs) are emerging as a promising technological direction to reduce cost in optical networks. In FONs, Reconfigurable Optical Add/Drop Multiplexers (ROADM), based on costly Wavelength Selective Switches (WSS), are replaced by broadcast-and-select switching architectures composed by passive splitters and combiners, which interconnect add/drop nodes via fiber trees [1]. However, due to channels' broadcast on unnecessary fiber links, FONs suffer a reduced spectral efficiency. Moreover, when amplifiers are deployed in FONs, the absence of WSSs causes propagation of Amplified Spontaneous Emission (ASE) noise beyond lightpath termination, and accumulation of ASE generated before a lightpath is initiated. Hence, FON architectures may become more sensitive to lightpaths degradation due to higher ASE noise compared to ROADM-based architectures (note that ROADMs contain WSSs, i.e., filters, that block unintended ASE accumulation). FONs have been already standardized and deployed as shown in [2]. In particular, FON deployment has attracted particular interest for metro networks [3] based on ring/horseshoe topologies. In metro, network operators, in the attempt to minimize network cost, are more prone to consider node architectures without WSSs, especially taking into account the high number of nodes in a metro network. Moreover, taking advantage of relatively short metro distances, operators have the opportunity to optimize also the number (and location) of Optical Amplifiers (OAs) needed to guarantee lightpaths Quality-of-Transmission (QoT). Hence, an optimized deployment of amplifiers is crucial to reduce network cost and maintain lightpaths feasibility in FONs. An optimized deployment must consider different amplifier types, i.e., boosters, pre-amplifiers and in-line amplifiers (ILAs), considering their different characteristics in terms of gain and noise figure.

In this letter, we investigate how to minimize the cost of OAs in FONs, while maintaining lightpath feasibility, expressed as a minimum requirement in terms of end-toend OSNR and optical power at the receiver. We propose a Genetic Algorithm (GA) to perform OSNR-aware OA placement which considers the differences between boosters, preamplifiers and ILAs. Although the problem of OA placement has received attention for ROADM-based networks [4], [5], to the best of our knowledge, no study has been done in the context of filterless metro networks, where the presence of ASE accumulation makes solutions for OA cost minimization (as the one in [4], [5]) not applicable.

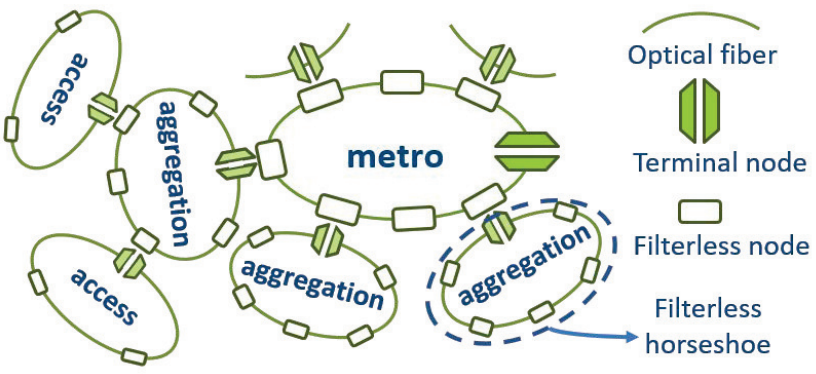

Fig. 1. Metro network composed of interconnected horseshoe topologies

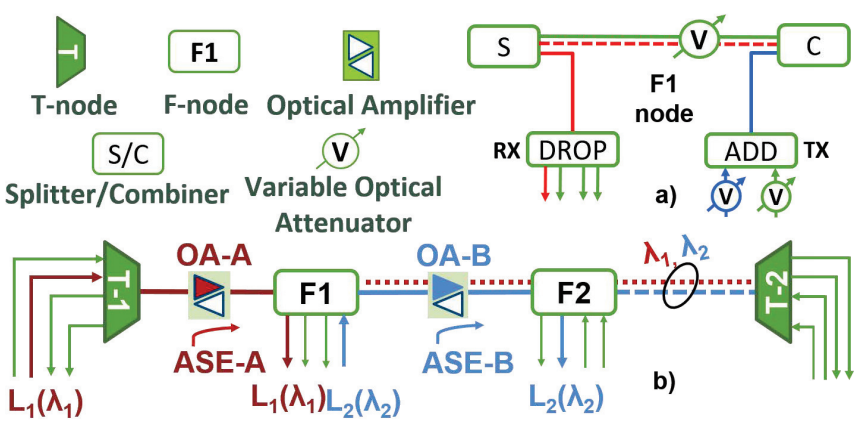

Fig. 2. a) Filterless node architecture and b) Example of channel broadcast and ASE accumulation in a filterless horseshoe topology

Note that while in [5] the OA placement is done through an iterative greedy-approach; the GA ensures a dynamic exploration of teh search-space. Moreover, considering FON nodes implies adapting the physical layer modeling to account for the ASE noise propagation beyond lightpath termination. 
We consider a practical case of a metro network composed by several interconnected filterless branches, where each branch is constituted by a horseshoe as shown in Fig. 1. These horseshoe topologies are becoming popular in metro aggregation networks [6] and typically contain two types of nodes: Terminal nodes (T-nodes) and Filterless nodes (F-nodes). T-nodes are interconnected to the rest of the metro network and equipped with filters that impede ASE noise propagation beyond them, and thus avoid creating laser loop effects due to the continuous propagation and amplification of the optical signal [7]. F-nodes that are placed along the optical line are equipped with passive splitters, combiners and Variable Optical Attenuators (VOAs) ${ }^{1}$. Fig. 2a shows the architecture of the filterless nodes considered in our study. To show the impact of ASE accumulation in a filterless horseshoe, consider the example in Fig. 2b. Two lightpaths are established on two different wavelengths, i.e., L1 between nodes $\mathrm{T} 1$ and $\mathrm{F} 1$ on $\lambda_{1}$, and L2 between nodes F1 and F2 on $\lambda_{2}$. The OA placed between nodes T1 and F1 (i.e., OA-A) introduces an ASE noise contribution (ASE-A), which propagates also beyond the filterless node $\mathrm{F} 1$ (dotted line) and accumulates with ASE-B generated by OA-B. Therefore, the overall ASE noise contribution (ASE-A and ASE-B) will propagate up to node $\mathrm{T} 2$ and, in particular, will affect the OSNR of lightpath L2.

\section{OPTICAL AMPLIFIER PLACEMENT IN FILTERLESS NETWORKS}

\section{A. Problem statement}

The problem of OA placement in filterless metro networks can be stated as follows: Given a horseshoe FON, a set of traffic demands (each characterized by source/destination nodes) and a set of candidate locations to place OAs, decide the OA placement (location ,i.e., booster, pre-amplifier and ILA) and decide the route and spectrum allocation (RSA) for each traffic demand, constrained by a required QoT for each lightpath (OSNR and received power thresholds), spectrum continuity and contiguity constraint, network capacity constraint, with the objective of minimizing the overall cost, constituted by the deployed OAs.

\section{B. Genetic Algorithm for OA placement}

Due to high combinatorial complexity of the problem (considering $x$ OA candidate locations, there are $2^{x}$ combinations of OA placement) and, its non-linear nature which requires to consider several cross-layer design parameters, we have developed a Genetic Algorithm (GA) to solve this problem. The evolutionary process of the GA is driven by competition among members (solutions) of the population and genetic operations, such as mutation and crossover. Each solution of the population is encoded as a string of binary values, i.e., the genes, which represent the candidate locations for OA placement, and assume value " 1 " if an OA is placed and " 0 " if the OA is not placed. In Fig.3a, we show an example of encoding the placement of OAs for two members of the

\footnotetext{
${ }^{1}$ The VOAs match/attenuate the channel powers according to the channel with the lower power level, thus regulate the power of optical channels.
}

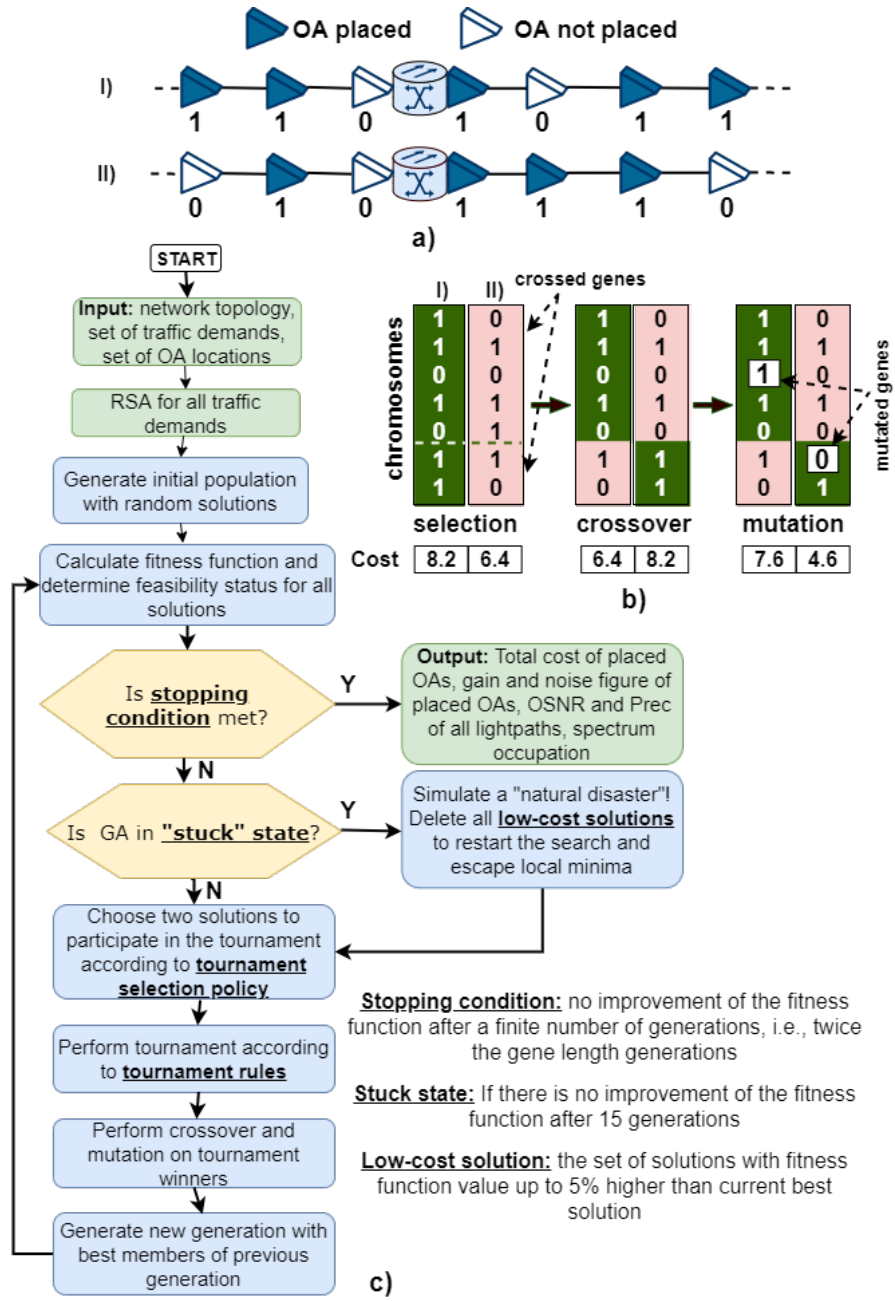

Fig. 3. a) Encoding of OA placement in a string of $1 \mathrm{~s}$ and $0 \mathrm{~s}$; b) crossover and mutation operations and c) flowchart of the proposed solution for optimized OA placement

population, noted as I) and II). We show the two members (chromosomes) as strings of $1 \mathrm{~s}$ and $0 \mathrm{~s}$ in Fig.3b (selection). A crossover point (dashed line) is randomly selected and the latter parts of the two members are swapped (crossover). A gene for each member is randomly selected and its value is inverted (mutation). Note that a cost value is reported for each member and the cost change due to crossover and mutation is reflected in each step. Each solution is characterized by its fitness value and feasibility status. Let us now define the fitness and feasibility of a solution: The fitness of a member of the population is determined by a cost function accounting for the type (set $T$ ) and location (set $L$ ) of the placed OAs. We define the fitness of a solution as the sum of costs of placed OAs:

$$
\text { fitness }=\sum_{l \in L} \sum_{t \in T}\left(O A_{l} * C_{l, t}\right)
$$

where $O A_{l}$ is a binary variable equal to 1 if an $\mathrm{OA}$ is placed in location $l$ and 0 otherwise, while $C_{l, t}$ is the cost of an OA of type $t$ placed in location $l$ (OA costs are reported in Sec.III). A lower fitness value is desirable as we minimize the total cost of OAs. Feasibility represents the extent to which a solution satisfies the constraints and is a real number between 0 and 1 . We define feasibility $f$ as the ratio between the total number 
of lightpaths $(L P)$ that are feasible (set $J$ ) to the total number of lightpaths routed (set $I$ ):

$$
f=\sum_{j \in J} L P_{j} / \sum_{i \in I} L P_{i}
$$

A lighthpath $i$ is feasible if it meets the QoT requirements, i.e., the total OSNR (which also comprises the non-linear noise from the GN-model [8]) and received power $\left(P_{r e c}\right)$ constraints $\left(O S N R_{i}>\right.$ threshold and $P_{r e c, i}>$ threshold, as defined in Eq. (5) and (6) in [5]). A solution is feasible if all lightpaths meet QoT requirements (thus $f=1$ ), whereas a solution is unfeasible if there is at least one lightpath in set $I$ which does not meet the OSNR and/or $P_{\text {rec }}$ constraint $(f<1)$.

In Fig. $3 \mathrm{c}$ we show the flowchart of the proposed solution, which performs RSA of traffic demands first and then OA placement. In the following, we elaborate on the main building blocks of the GA (highlighted text in bold), namely the Tournament selection policies, Tournament rules, "stuck" state and stopping condition.

The GA starts the search by generating an initial population as a random set of solutions while new populations are generated according to tournament selection policies. Tournament selection policies define the pairs of solutions to participate in the tournament. If no feasible solution has been found yet, the solution which provides the highest number of feasible demands is defined as the current best solution. Until no feasible solution has been found, members of the population are sorted in two groups: A) solutions close to current best solution and $B$ ) all other solutions. The set of solutions in group $A$ depends on how close is the current best solution to reach feasibility of all lightpaths (i.e., $f=1$ ). For example, if the current best solution has a $f=0.7$, then $5 \%$ of the best solutions are sorted in group $A$ or if $f=0.9$, then $1 \%$ of the best solutions are sorted in group $A$. In principle, the closer to a feasible solution, i.e., $f=1$, the lower is the $\%$ of best solutions sorted in group $A$. We consider solutions from group $A$ to participate in the tournament $70 \%$ of the time, whereas solutions from group $B, 30 \%$ of the time.

Once the GA starts finding feasible solutions, members of the population are sorted in three groups: $A$ ) feasible solutions with a low fitness value, $B$ ) feasible solutions with a high fitness value $C$ ) unfeasible solutions with a $f>0.95$ and a low fitness value. We consider a solution to have a low (high) fitness value if it is not 5\% (25\%) higher than the best solution found so far. Solutions from group $A$ participate in the tournament $70 \%$ of the time, solutions from group $B, 20 \%$, and solutions from group $C, 10 \%$ of the time. We have fine-tuned the tournament selection policies based on the performance of the GA so that it converges quickly, i.e., in a few generations. Tournament rules are defined as follows: $i$ ) a feasible solution wins over an unfeasible solution; ii) if two feasible solutions compete, the solution with the lower fitness wins and iii) if two unfeasible solutions compete, the winner is chosen at random. New generations are created by performing genetic operations (crossover and mutation) on tournament winners. In case there is no improvement of the fitness function after a finite number of generations, we consider the GA to be in a "stuck" state. In this case, all feasible solutions which have a low fitness value are deleted in order to restart the search process and try to escape the local minimum. If there is no improvement of the fitness value for 15 generations, the GA reaches a local optimum, i.e., "stuck" state. The stopping condition for the GA is reached if there is no improvement for a number of generations equal to twice the number of genes. Therefore, if the GA has reached the "stuck" state and there is no improvement for twice the number of genes generations, then the GA stops the search. Note that the GA may find several best-cost solutions, however, in our results we show only the solution with the highest minimal OSNR (minOSNR).

We propose two versions of the GA, minCostGA and ConstrainedGA. Since the objective of $\operatorname{minCost} G A$ is to minimize cost, this may lead to a drawback on the OSNR performance. ConstrainedGA overcomes the drawbacks of minCostGA and aims at minimizing the OA cost while guaranteeing the OSNR performance provided by the benchmark approaches. While $\min \operatorname{Cost} G A$ is constrained to a minOSNR equal to $13 \mathrm{~dB}$, ConstrainedGA is constrained by the minOSNR achieved by the benchmark approach, i.e., OSNR threshold is set equal to minOSNR of benchmark.

\section{Illustrative Numerical RESUlts}

In our numerical evaluation, we compare different technologies (FON vs WSON) considering horseshoe topologies of different lengths and with different number of nodes. As FON and WSON have different node architectures, T-nodes, considered in WSON are assumed to introduce a $17 \mathrm{~dB}$ loss whereas F-nodes, considered in FON introduce a $13 \mathrm{~dB}$ loss (additional $4 \mathrm{~dB}$ loss in T-nodes may lead to deploy more OAs in WSON compared to FON containing F-nodes). Note that the add/drop path introduces half of the node loss, e.g., 6.5 $\mathrm{dB}$ loss in FON. We model optical nodes loss as an equivalent fiber span loss generated in a fiber with length equal to $68 \mathrm{~km}$ and $52 \mathrm{~km}$ for T-nodes and F-nodes, respectively. Note that, we consider that no non-linear interference (NLI) is generated in the fiber span mimicking the node. We assume Standard Single-Mode Fiber (SSMF) with attenuation coefficient of 0.25 $\mathrm{dB} / \mathrm{km}$. We consider the placement of two types of OAs that differ in terms of gain ${ }^{2}(\mathrm{G})$ and noise figure (NF). Booster OAs are characterized by a $\mathrm{G}$ range of (10-20) $\mathrm{dB}$ and a $\mathrm{NF}$ range of (6-12) $\mathrm{dB}$, while pre-amp OAs by a $\mathrm{G}$ range of (18-32) dB and a NF range of (6.2-10.5) $\mathrm{dB}^{3}$. We consider the placement of OAs in three possible locations: $i$ ) at the ingress of the node (for pre-amplifiers), ii) at the egress of the node (for boosters) and iii) along the fiber (for inline amplifiers, which have the same characteristics of boosters). We assume the cost of a booster is $1 \mathrm{cu}$ (cost unit) while the cost of a pre-amplifier is $1.2 \mathrm{cu}$. If deployed as an ILA, the cost of an OA assumes an additional $0.8 \mathrm{cu}$ accounting for the deployment, maintenance and management of the cabinet/location (e.g., therefore, the cost of a booster type deployed as an ILA is $1.8^{4}$ ). In all our

\footnotetext{
${ }^{2} \mathrm{We}$ assume a flat OA gain profile due to the use of gain flattening filters. ${ }^{3}$ Note that a high NF corresponds to a low $\mathrm{G}$ and vice-versa.

${ }^{4} \mathrm{SM}-$ Optics, private communication
} 
TABLE II

TABLE I

\begin{tabular}{ccc}
\multicolumn{3}{c}{ Cost of GA vs BF $[\mathrm{cu}]$} \\
\hline CL & GAcost & BFcost \\
\hline 24 & 18.0 & 18.0 \\
30 & 18.6 & 18.0 \\
36 & 18.6 & 18.0 \\
\hline
\end{tabular}

MINIMUM (AVERAGE) OSNR VALUES IN [DB] FOR THE 6-NODE HORSESHOE TOPOLOGY

\begin{tabular}{cccccc}
\hline Strategy & $\mathbf{1 0 0} \mathbf{~ k m}$ & $\mathbf{3 0 0} \mathbf{~ k m}$ & $\mathbf{5 0 0} \mathbf{~ k m}$ & $\mathbf{7 0 0} \mathbf{~ k m}$ & $\mathbf{9 0 0} \mathbf{~ k m}$ \\
\hline minCostGA-FON & $19.24(25.89)$ & $14.05(18.02)$ & $13.39(16.13)$ & $13.52(15.73)$ & $13.41(15.46)$ \\
ConstrainedGA-FON & $22.73(28.32)$ & $20.39(23.70)$ & $19.22(22.50)$ & $18.49(21.54)$ & $17.89(20.83)$ \\
minCostGA-WSON & $16.15(24.57)$ & $13.99(20.45)$ & $13.54(18.22)$ & $13.36(17.71)$ & $13.44(17.57)$ \\
CostrainedGA-WSON & $21.68(27.03)$ & $19.98(24.47)$ & $18.83(23.32)$ & $18.17(22.68)$ & $17.66(22.01)$ \\
Baseline-FON & $21.40(23.59)$ & $20.11(22.34)$ & $18.91(21.15)$ & $18.15(20.39)$ & $17.57(19.83)$ \\
Baseline-WSON & $21.15(25.09)$ & $19.74(23.73)$ & $18.64(22.64)$ & $17.93(21.94)$ & $17.39(21.41)$ \\
\hline
\end{tabular}

is varied, simulating small metro $(100 \mathrm{~km})$ and regional metro $(900 \mathrm{~km})$ networks. Results are averaged for each horseshoe length by considering 20 topologies with random link lengths with a variability of $+/-50 \%$ from the average length of a link. For example, a horseshoe of length $300 \mathrm{~km}$ with 5 links, the average link length is $60 \mathrm{~km}$ so each link's length is equal to a random value between $30 \mathrm{~km}$ and $90 \mathrm{~km}$. For these two network case studies, we compare five approaches: the $\min \operatorname{Cost} G A$ (i.e., minCostGA-FON and minCostGA-WSON in the figure) and ConstrainedGA (i.e., Constrained GA-FON and ConstrainedGA-WSON in the figure) against the Baseline OA placement. Recapping: $\min \operatorname{Cos} G A$, finds the minimum-cost solution and represents a lower-bound in terms of the cost of OAs while ensuring that the $\operatorname{minOSNR}$ of the lightpaths meets the required threshold. ConstrainedGA, aims at minimizing OA cost while guaranteeing minimal OSNR values for all lightpaths which are higher or equal to the values provided by the Baseline strategy.

As a benchmark strategy, we consider a baseline OA placement (intended to represent current OA placement strategy) working as follows: $i$ ) all nodes are equipped with preamplifiers and boosters (booster OA gain is set to compensate for the node loss and pre-amp gain set to compensate for the span it terminates), and ii) inline amplifiers are placed every $60 \mathrm{~km}$ (considering OA gain set to compensate span losses, this corresponds to gain of $15 \mathrm{~dB}$ in all ILAs). In Fig. 4 we report the results obtained for the two topologies, showing the OA cost (in $\mathrm{cu}$ ) for the cases of filterless network (FON) and WSS-based wavelength-switched optical network (WSON).

minCostGA vs Baseline: Results show significant savings in total OA cost achieved by GA in comparison to the baseline approach ${ }^{5}$. Specifically, the savings range between $45 \%$ and $60 \%$ for the 6-node topology and between $45 \%$ and $59 \%$ for the 11-node topology for the filterless horseshoe scenario ( $\min \operatorname{Cos} t G A-F O N$ vs Baseline). Similarly, savings for minCostGA-WSON compared to Baseline between $42 \%$ to $52 \%$ for the 6-node topology and between $35 \%$ and $55 \%$ for the 11-node topology are achieved. These results confirm that relevant OA cost savings can be achieved in metro filterless networks. Even though $\min \operatorname{Cost} G A$ provides significant savings in total OA cost, OSNR performance of minCost $G A$ is significantly worse compared to Baseline.

Tab. II shows the minimal and average OSNR of lightpaths for the 6-node topology considering $\min \operatorname{Cost} G A-F O N$, $\min \operatorname{Cost} G A-W S O N$ and the Baseline-FON and Baseline-

\footnotetext{
${ }^{5}$ Note that Baseline deployment is the same for FON and WSON
} 
WSON. We observe that Baseline-FON provides a better OSNR performance with a minOSNR (avgOSNR) up to 6.06 (5.02) dB higher compared to minCostGA-FON. Similarly, Baseline-WSON provides a higher minOSNR (avgOSNR) up to 5.76 (4.42) $\mathrm{dB}$ compared to minCostGA-WSON.

1) ConstrainedGA vs Baseline: We now observe the performance of ConstrainedGA that still targets OA cost savings, but constraining the GA in such a way that OSNR is guaranteed to be at least as in the Baseline case.

Fig. 4 shows that ConstrainedGA-FON achieves cost savings ranging between $31 \%$ and $56 \%$ for the 6-node topology and between $32 \%$ and $59 \%$ for the 11-node topology compared to Baseline-FON. Similarly, ConstrainedGA-WSON achieves cost savings between $26 \%$ and $41 \%$ for the 6-node topology and between $19 \%$ and $54 \%$ for the 11-node topology, compared to Baseline-WSON. The minimal and average OSNR values for ConstrainedGA shown in Tab. II are now higher compared to Baseline achieving higher values up to 1.33 (4.73) $\mathrm{dB}$ in terms of minOSNR (avgOSNR) in FON and 0.53 (1.94) $\mathrm{dB}$ in WSON. In conclusion, ConstrainedGA is capable of achieving significant $\mathrm{OA}$ cost savings while ensuring higher OSNR compared to baseline OA deployment.

2) FON vs WSON: Let us now observe the different deployment of OAs for FON vs WSON. In terms of total cost of OAs, minCostGA-FON ensures savings between $6 \%$ and $21 \%$ compared to $\min \operatorname{Cost} G A-W S O N$ for the 6-node topology and between $10 \%$ and $21 \%$ for the 11-node topology. ConstrainedGA-FON ensures savings between $7 \%$ and $25 \%$ compared to ConstrainedGA-WSON for the 6-node topology and between $10 \%$ and $30 \%$ for the 11-node topology. These results confirm that FON leads to a significant reduction in total OA cost in metro networks.

Regarding the minimal and average OSNR of lightpaths for the 6-node topology, results for FON vs WSON are shown in Tab. II. We observe that: i) minOSNR is lower in WSON compared to FON and ii) avgOSNR is lower in FON compared to WSON. AvgOSNR is lower in FON compared to WSON because ASE noise accumulation has a higher impact in FON, i.e., more lightpaths are affected by the propagated ASE noise beyond lightpath termination. Instead, minOSNR is lower in WSON because minOSNR is experienced on the longest path, i.e., the path between the two furthest nodes in the case of a horseshoe, for this path, the ASE noise and NLI contributions are the same along the fiber spans (if the OA placement is the same, e.g., Baseline). However, the higher node loss in WSON leads to a higher ASE noise generated by the OA following the node, e.g., booster OA, therefore, the higher node loss in WSON is the decisive factor why minOSNR is lower in WSON compared to FON.

Finally, we confirm that, due to the channel broadcast feature, FONs has a higher spectrum occupation in comparison to WSONs. Specifically, FONs occupy $36 \%$ (43\%) more spectrum compared to WSONs for the 6-node (11-node) topology. C. Sensitivity analysis: OA Gain Ripple

We perform a sensitivity analysis on the robustness of solutions provided by GA, accounting for the unpredictable
TABLE III

PROBABILITY $(p)$ OF MINOSNR BEING BELOW 13 DB DUE TO OA GAIN RIPPLE: P(MINOSNR <13DB) FOR 6-NODE 900 KM AND $500 \mathrm{KM}$ TOPOLOGIES CONSIDERING MINCOSTGA, CONSTRAINEDGA AND BASELINE STRATEGIES

\begin{tabular}{cccc}
\hline Strategy (horseshoe km) & Cost [cu] & minOSNR [dB] & p \\
\hline minCostGA (900) & 38 & 13.12 & 0.68 \\
constrainedGA (900) & 50.8 & 17.76 & 0.00 \\
Baseline (900) & 68.8 & 17.36 & 0.00 \\
minCostGA (500) & 22.6 & 13.71 & 0.26 \\
constrainedGA (500) & 31.2 & 18.81 & 0.00 \\
Baseline (500) & 43.6 & 18.78 & 0.00 \\
\hline
\end{tabular}

variation due to OA gain ripple. We assume a uniform statistical variation in the $\mathrm{OA}$ gain with a variance of $1.5 \mathrm{~dB}$ and $1 \mathrm{~dB}$ for pre-amp and booster type, respectively. Provided the solution by the GA, we consider the variance of the OA gain a posteriori and repeat this simulation 1000 times in order to achieve a statistical confidence. For each GA solution we determine the probability that a solution is unfeasible due to OA gain variation. In Tab. III we report the probability (p) that minOSNR is below the OSNR threshold (13 dB) for the 6-node $500 \mathrm{~km}$ and $900 \mathrm{~km}$ topology for minCostGA, constrainedGA and Baseline solutions. While $\mathrm{p}$ is equal to 0.26 and 0.68 for $500 \mathrm{~km}$ and $900 \mathrm{~km}$ for minCostGA, constrainedGA and Baseline have a $\mathrm{p}$ equal to zero for both cases. The constrainedGA solutions not only achieve significant cost savings and meet the OSNR performance of the Baseline but also ensure that random fluctuations of OSNR due to gain ripple do not lead to unfeasible solutions. Due to page limitation, we do not report the analysis for other horseshoe lengths, however, we note that for $100 \mathrm{~km}$ and 300 $\mathrm{km}$ also minCostGA achieves $\mathrm{p}$ equal to zero.

In conclusion, we propose two versions of a Genetic Algorithm (i.e., minCostGA and Constrained $G A$ ) for OA placement in FON and WSON metro topologies and compare their performance against a Baseline OA deployment. We show that minCostGA can achieve up to $60 \%$ (55\%) OA cost savings compared to Baseline for FON (WSON). Moreover, we show that ConstrainedGA achieves up to $59 \%$ (54\%) OA cost savings for FON (WSON) while guaranteeing better OSNR performance compared to Baseline.

\section{REFERENCES}

[1] C. Tremblay, et al. "Agile Optical Networking: Beyond Filtered Solutions," in OFC, San Diego, CA, 2018, paper M1A.5.

[2] M. Gunkel, et al. "Vendor-interoperable elastic optical interfaces: Standards, experiments, and challenges" in JOCN, vol. 7, no. 12, pp. B184B193, 2015.

[3] P. Pavon-Marino, et al. "Techno-economic impact of filterless data plane and agile control plane in the 5G optical metro" in JLT vol.38, n.15, pp.3801-3814, 2020.

[4] J. Pedro, et al. "Optimized Hybrid Raman/EDFA Amplifier Placement for DWDM Mesh Networks" in JLT vol. 36, n. 9, pp. 1552-1561, 2018.

[5] M. Ibrahimi, et al. "Minimum-Cost Optical Amplifier Placement in Metro Networks," in JLT vol. 38, n. 12, pp. 3221-3228, 2020.

[6] F. Paolucci, et al. "Disaggregated edge-enabled C+L-band filterless metro networks," in JOCN vol. 12, n. 3, pp. 2-12, 2020.

[7] C. Tremblay, et al. "Passive filterless core networks based on advanced modulation and electrical compensation technologies" in Telecommunication Systems vol. 54, no. 2, pp. 167-181, 2013.

[8] P. Poggiolini, et al."The GN-Model of Fiber Non-Linear Propagation and its Applications," in JLT vol. 32, no. 4, pp. 694-721, 2014. 